\title{
Long-term outcomes and prognostic factors with non-curative endoscopic submucosal dissection for gastric cancer in elderly patients aged $\geq 75$ years
}

\author{
Yosuke Toya ${ }^{1}$ - Masaki Endo ${ }^{1,2} \cdot$ Shotaro Nakamura $^{1} \cdot$ Risaburo Akasaka $^{1} \cdot$ Shunichi Yanai $^{1} \cdot$ Keisuke Kawasaki $^{1}$. \\ Keisuke Koeda ${ }^{3} \cdot$ Makoto Eizuka $^{4} \cdot$ Yasuko Fujita ${ }^{4} \cdot$ Noriyuki Uesugi $^{4} \cdot$ Kazuyuki Ishida $^{4} \cdot$ Tamotsu Sugai $^{4}$. \\ Takayuki Matsumoto ${ }^{1}$
}

Received: 16 April 2018 / Accepted: 11 December 2018 / Published online: 17 December 2018

(c) The International Gastric Cancer Association and The Japanese Gastric Cancer Association 2018

\begin{abstract}
Background Little is known about the long-term outcomes and prognostic factors with non-curative endoscopic submucosal dissection (ESD) in elderly patients with early gastric cancer.

Methods Clinicopathological findings and long-term outcomes were evaluated in 87 patients with early gastric cancer (EGC) aged $\geq 75$ years who were treated with non-curative ESD. Prognostic factors for overall survival (OS) were analyzed with the Kaplan-Meier method and a Cox proportional hazards model.

Results During the follow-up period, among 27 patients who died of any cause, only one patient died of gastric cancer. OS probabilities after 3 and 5 years were $89.7 \%$ and $79.3 \%$, respectively. Univariate analyses revealed that Eastern Cooperative Oncology Group performance status $2-3$, Charlson comorbidity index $(\mathrm{CCI}) \geq 3$, neutrophil/lymphocyte ratio $\geq 3.3$, prognostic nutritional index $<44.8$, distal tumor location and macroscopically depressed or flat configuration were associated with poor OS. Cox multivariate analysis revealed high CCI $(\geq 3)$ to be an independent prognostic factor associated with OS (hazard ratio: $2.63,95 \%$ confidence interval [CI] 1.06-6.49, $P=0.037$ ).

Conclusions CCI may be a useful parameter for decision-making regarding additional surgery for elderly patients with gastric cancer treated by non-curative ESD.
\end{abstract}

Keywords Gastric cancer · Elderly patients · Prognostic factors $\cdot$ Charlson comorbidity index

\section{Introduction}

In recent decades, the size of the elderly population has been increasing rapidly worldwide. Gastric cancer is still a frequent cause of death in Japan. Endoscopic submucosal

Yosuke Toya

ytoya@iwate-med.ac.jp

1 Division of Gastroenterology, Department of Internal Medicine, School of Medicine, Iwate Medical University, Uchimaru 19-1, Morioka 020-8505, Japan

2 Kaiunbashi Endoscopy Clinic, Morioka, Japan

3 Department of Surgery, School of Medicine, Iwate Medical University, Morioka, Japan

4 Division of Molecular Diagnostic Pathology, Department of Pathology, School of Medicine, Iwate Medical University, Morioka, Japan dissection (ESD) is an established, standard treatment for early gastric cancer (EGC) [1-5]. ESD is accepted as a less invasive treatment and provides improvement in postoperative quality of life compared to open surgery $[6,7]$. In addition, a number of studies have revealed excellent short and long-term outcomes after ESD for EGC, even in elderly patients [8-14]. Due to the increasing necessity of ESD for elderly patients, however, physicians are facing the problem of how to decide whether to perform additional gastrectomy in elderly patients with EGC who undergo non-curative ESD.

To date, several studies have reported the clinical outcomes of EGC cases after non-curative ESD [15-19]. However, only a few studies have reported the long-term outcomes with non-curative ESD in elderly patients. In addition, elderly patients have higher rates of all-cause mortality than younger patients. The aim of this study was to clarify 
the long-term outcomes and prognostic factors with noncurative ESD for EGC in elderly patients.

\section{Methods}

\section{Patients}

We performed ESD for 1358 patients with EGC at our institute between June 2002 and December 2012. Among these 1358 patients, 39 patients with remnant stomachs, 4 patients with gastric tubes, and 103 patients of unknown status were excluded. Of the remaining 1212 patients, 1008 patients who met the curability criteria were excluded. Of the remaining 204 patients, 87 patients aged $\geq 75$ years were the subjects of the present study (Fig. 1).

Patient medical charts were reviewed to obtain data on clinical and demographic characteristics, including age, gender, Eastern Cooperative Oncology Group (ECOG) performance status (PS) [20], and body mass index (BMI). We evaluated the following items as possible prognostic factors: Geriatric Nutritional Risk Index (GNRI) [21, 22], Charlson comorbidity index (CCI) [23], neutrophil to lymphocyte ratio (NLR) [24] and prognostic nutritional index (PNI) [25]. The GNRI was calculated with the values of serum albumin and BMI listed on patient medical charts just before ESD: GNRI $=14.89 \times$ serum albumin $(\mathrm{g} / \mathrm{dL})+41.7 \times \mathrm{BMI} / 22$ [22]. The CCI was calculated with scores based on the original definition [23]. The NLR was calculated by dividing the total neutrophil count by the total lymphocyte count [24]. The PNI was calculated with the serum albumin level and the total lymphocyte count: $\mathrm{PNI}=10 \times$ serum albumin $(\mathrm{g} /$ $\mathrm{dL})+0.005 \times$ total lymphocyte count (per $\mathrm{mm}^{3}$ ) [25]. Written informed consent was obtained from all patients before ESD. This study protocol was approved by the ethical committee of Iwate Medical University (H29-182).

\section{Curability criteria}

Curability of ESD was determined on the basis of guidelines reported by the Japanese Gastric Cancer Association [26] and the Japanese Gastroenterological Endoscopy Association [27]. When a lesion was resected en bloc, was $<2 \mathrm{~cm}$ in diameter, and was predominantly a differentiated type, pathologically intramucosal carcinoma (pT1a), lacking in ulcerative findings (UL[-]) and lymphovascular invasion (ly0, v0), with negative surgical margins (R0), the procedure was considered a curative resection by absolute indication. When a lesion was resected en bloc and was (1) $\geq 2 \mathrm{~cm}$ in diameter, predominantly a differentiated type, pT1a, and $\mathrm{UL}(-) ;(2)<3 \mathrm{~cm}$, predominantly a differentiated type, pT1a, and UL $(+) ;(3)<2 \mathrm{~cm}$, predominantly an undifferentiated type, pT1a, and UL(-); or $(4)<3 \mathrm{~cm}$, predominantly a differentiated type, pathologically minute submucosal $(\mathrm{SM})$ cancer $<500 \mu \mathrm{m}$ (pT1b/SM1) in vertical depth, and with negative surgical margins (R0), the procedure was considered a curative resection by the expanded indications. When a lesion did not meet the criteria of the absolute or the expanded indications, the procedure was considered a noncurative resection, as described previously [19].

\section{Follow-up and collection of outcome data}

The selection of either additional gastrectomy or follow-up without gastrectomy was determined by the chief physician for each patient in consideration of the risk of gastrectomy. For patients who underwent additional gastrectomy, endoscopic examination was conducted once a year, and computed tomography (CT) was performed 6 months after gastrectomy, and thereafter once a year. For patients who were followed without gastrectomy, endoscopic examination was conducted 1-3 months after ESD. Helicobacter pylori $(H$.
Fig. 1 Flow of patients enrolled in the study

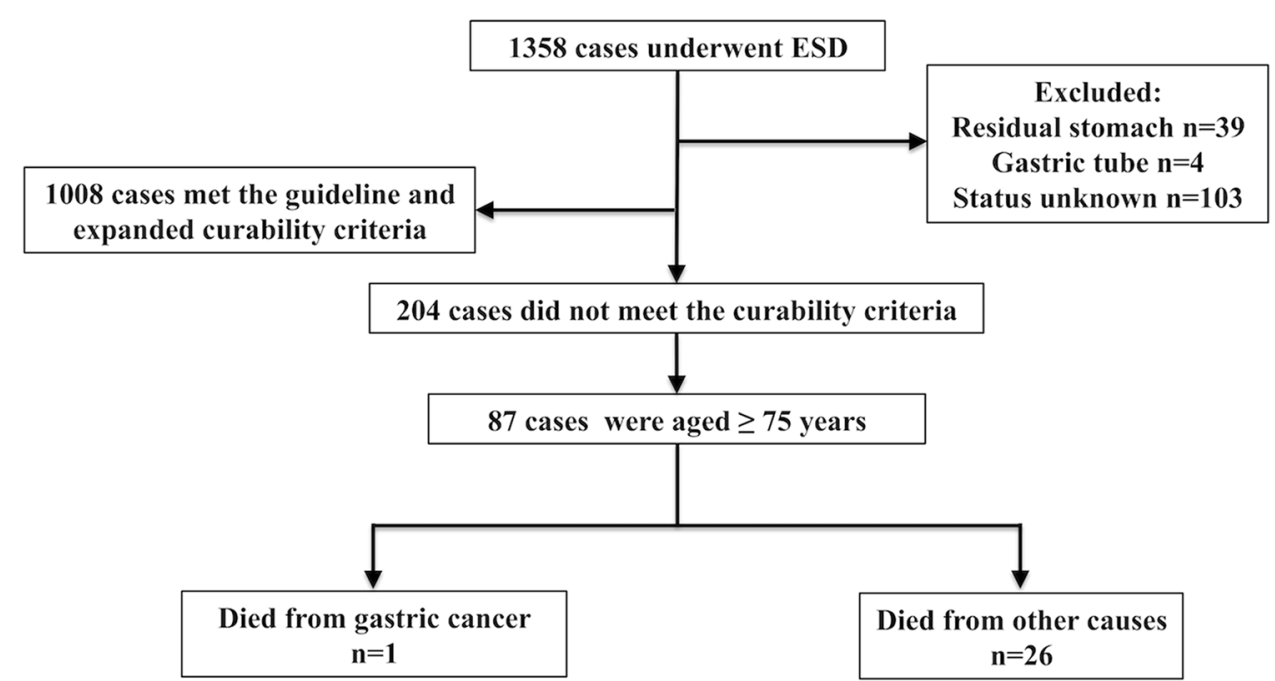


pylori) was eradicated in infected patients. Thereafter, endoscopic examinations were conducted at 6 and 12 months. Unless local recurrence was found, yearly endoscopic examination was continued. Abdominal ultrasound and CT were also performed once a year. For patients who were followed up outside of our institution, we conducted an annual questionnaire survey via their primary care physicians. If patients had not made hospital visits, we contacted them or their family members directly to confirm the prognosis.

\section{Statistical analysis}

Overall survival (OS) after ESD was analyzed with the Kaplan-Meier method, and the differences between groups were assessed by the log-rank test. The relationship between OS and each clinicopathologic factor was analyzed by univariate analysis with the log-rank test. Cut-off values for the GNRI were determined based on a previous report [21]. Cut-off values for the NLR and PNI were determined by receiver operating characteristic (ROC) analysis. Values that maximized the sensitivity and specificity for 5-year OS were used as the cut-off values. Possible prognostic factors were included in multivariate analyses with a Cox proportional hazards regression model. A $P$ value $<0.05$ was considered to be statistically significant. All of the statistical analyses were performed with SPSS version 22 software for MAC OS (SPSS Inc., Chicago, IL, USA) and JMP version 12 (Statistical Discovery Program, Cary, NC, USA).

\section{Results}

Table 1 shows the demographic and clinical characteristics of the study population. The median age was 78 years, and most patients were male (74.7\%). The ECOG PS was 0 or 1 in 66 patients (75.9\%). The median BMI was $22.7 \mathrm{~kg} / \mathrm{m}^{2}$, and the median follow-up period was 6.7 years. The mean GNRI was 103.4, and 66 patients (75.9\%) had a CCI of $0-2$. The median NLR was 2.3, and the mean PNI was 48.1.

The clinicopathological characteristics of the resected EGCs are summarized in Table 2. Of the 87 tumors, 81 (93.1\%) were initial cancers and $6(6.9 \%)$ were metachronous cancers. The most frequent location was the middle third portion of the stomach, and the median tumor size was $23 \mathrm{~mm}$. Most tumors were the histologically differentiated type (92\%), and 24 tumors (27.6\%) had invaded the deep portion of the submucosa. Lymphovascular invasion was positive in 26 tumors (29.9\%), and ulcerative findings were noted in 18 tumors $(20.7 \%)$. The median procedure time for ESD was $66 \mathrm{~min}$. As for adverse events, postoperative bleeding was observed in one $(1.1 \%)$ patient and perforation was observed in five $(5.7 \%)$ patients.
Table 1 Demographic and clinical characteristics of 87 patients aged $\geq 75$ years who underwent non-curative ESD for gastric cancer

\begin{tabular}{ll}
\hline Age, years, median (range, IQR) & $78(75-88,7)$ \\
Gender, $n(\%)$ & $65(74.7)$ \\
Male & $22(25.3)$ \\
Female & \\
ECOG PS, $n(\%)$ & $28(32.2)$ \\
0 & $38(43.7)$ \\
1 & $19(21.8)$ \\
2 & $2(2.3)$ \\
3 & $22.7(16.2-33.3,3.3)$ \\
Body mass index, kg/m ${ }^{2}$, median (range, IQR) & $6.7(0.1-14.8,3.8)$ \\
Follow-up period, years, median (range, IQR) & $103.4( \pm 9.7)$ \\
GNRI, mean ( \pm SD) & \\
CCI, $n(\%)$ & $30(34.5)$ \\
0 & $24(27.6)$ \\
1 & $12(13.8)$ \\
2 & $15(17.2)$ \\
3 & $3(3.4)$ \\
4 & $2(2.3)$ \\
5 & $1(1.1)$ \\
6 & $2.3(0.6-11.9,1.3)$ \\
NLR, median (range, IQR) & $48.1( \pm 5.3)$ \\
PNI, mean $( \pm$ SD) &
\end{tabular}

$E S D$ endoscopic submucosal dissection, $I Q R$ inter-quartile range, ECOG PS Eastern Cooperative Oncology Group performance status, $S D$ standard deviation, GNRI geriatric nutritional risk index, $C C I$ Charlson comorbidity index, $N L R$ neutrophil to lymphocyte ratio, $P N I$ prognostic nutritional index

Additional gastrectomy was performed in 26 patients (29.9\%), of whom 20 patients underwent distal gastrectomy, and 6 patients underwent total gastrectomy. The final histopathologic findings were as follows: 7 patients had residual cancer, and another patient had lymph node metastasis. In the remaining 19 patients, neither residual cancer nor lymph node metastasis was found.

During the follow-up period, recurrence of primary EGC was found in two patients. A patient was found to have local recurrence after ESD with positive horizontal margin. This patient was treated by additional ESD without subsequent recurrence. The other patient was treated by gastrectomy after ESD because of submucosal invasion and positive lymphatic permeation. The patient died of peritoneal dissemination of the primary EGC 2 years after the surgery.

Metachronous gastric cancers were observed in 8 patients (9.2\%). All 8 patients had been successfully treated with additional ESD. During the follow-up period, among 27 patients who died, only one patient died of gastric cancer. Probabilities of OS after 3 and 5 years were $89.7 \%$ and $79.3 \%$, respectively.

Results of univariate analyses for possible prognostic factors are summarized in Table 3. Patients who had ECOG PS 2-3, high CCI ( $\geq 3)$, high NLR $(\geq 3.3)$, low 
Table 2 Clinicopathological characteristics of 87 patients aged $\geq 75$ years who underwent non-curative ESD for gastric cancer

\begin{tabular}{|c|c|}
\hline \multicolumn{2}{|l|}{ Tumor type, $n(\%)$} \\
\hline Primary cancer & $81(93.1)$ \\
\hline Metachronous cancer & $6(6.9)$ \\
\hline \multicolumn{2}{|l|}{ Tumor location, $n(\%)$} \\
\hline Upper & $22(25.3)$ \\
\hline Middle & $42(48.3)$ \\
\hline Lower & $23(26.4)$ \\
\hline Tumor size, mm, median (range, IQR) & $23.0(3-95,22)$ \\
\hline \multicolumn{2}{|l|}{ Macroscopic appearance, $n(\%)$} \\
\hline Elevated & $46(52.9)$ \\
\hline Depressed/flat & $41(47.1)$ \\
\hline \multicolumn{2}{|l|}{ Histology, $n(\%)$} \\
\hline Differentiated type & $80(92.0)$ \\
\hline Undifferentiated type & $7(8.0)$ \\
\hline \multicolumn{2}{|l|}{ Depth of invasion, $n(\%)$} \\
\hline M/SM1 & $63(72.4)$ \\
\hline SM2 & $24(27.6)$ \\
\hline Lymphovascular invasion, $n(\%)$ & $26(29.9)$ \\
\hline Ulcerative findings, $n(\%)$ & $18(20.7)$ \\
\hline Procedure time, min, median (range, IQR) & $66(8-632,78)$ \\
\hline $\begin{array}{l}\text { Adverse events } \\
\text { Postoperative bleeding, } n(\%)\end{array}$ & $1(1.1)$ \\
\hline Perforation, $n(\%)$ & $5(5.7)$ \\
\hline Additional gastrectomy, $n(\%)$ & $26(29.9)$ \\
\hline Lymph node metastasis, $n(\%)$ & $1(1.1)$ \\
\hline Other metachronous cancers, $n(\%)$ & $8(9.2)$ \\
\hline $\begin{array}{l}\text { Prognosis } \\
\text { Death due to any causes, } n(\%)\end{array}$ & $27(31.0)$ \\
\hline Death due to gastric cancer, $n(\%)$ & $1(1.1)$ \\
\hline
\end{tabular}

$E S D$ endoscopic submucosal dissection, $I Q R$ inter-quartile range, $M$ mucosa, SM1 superficial portion of the submucosa within $500 \mu \mathrm{m}$ from the muscularis mucosae, SM2 deep portion of the submu$\operatorname{cosa} \geq 500 \mu \mathrm{m}$ from the muscularis mucosae, min minutes

PNI (<44.8), tumor location in the lower portion of the stomach and depressed/flat type of macroscopic configuration had worse OS than patients without those factors. Although the difference was not statistically significant, 5 -year OS was higher in patients with additional gastrectomy than in those without ( 0.85 vs $0.77, P=0.34)$.

Table 4 shows the results of multivariate analysis using a Cox proportional hazards model including possible prognostic factors. As shown in the table, only high CCI $(\geq 3)$ was found to be an independent prognostic factor associated with worse OS (hazard ratio, $2.79 ; 95 \% \mathrm{CI}$ $1.16-6.69 ; P=0.021)$. The overall survival rate was significantly lower in a high-CCI group than in a low-CCI group (Fig. 2, $P<0.001$ ).
Table 3 Overall survival (Kaplan-Meier method)

\begin{tabular}{|c|c|c|c|}
\hline Variable & No. of patients & 5-year OS & $P$ value \\
\hline \multicolumn{4}{|l|}{ Age } \\
\hline$\geq 79$ years & 41 & 0.73 & \multirow[t]{2}{*}{0.06} \\
\hline$<79$ years & 46 & 0.85 & \\
\hline \multicolumn{4}{|l|}{ Gender } \\
\hline Male & 65 & 0.77 & \multirow[t]{2}{*}{0.11} \\
\hline Female & 22 & 0.86 & \\
\hline \multicolumn{4}{|l|}{ ECOG PS } \\
\hline $0-1$ & 66 & 0.85 & \multirow[t]{2}{*}{0.001} \\
\hline $2-3$ & 21 & 0.62 & \\
\hline \multicolumn{4}{|l|}{ GNRI } \\
\hline$\geq 92$ & 79 & 0.78 & \multirow[t]{2}{*}{0.91} \\
\hline$<92$ & 8 & 0.75 & \\
\hline \multicolumn{4}{|l|}{$\mathrm{CCI}$} \\
\hline $0-2$ & 66 & 0.88 & \multirow[t]{2}{*}{$<0.001$} \\
\hline$\geq 3$ & 21 & 0.52 & \\
\hline \multicolumn{4}{|l|}{ NLR } \\
\hline$\geq 3.3$ & 13 & 0.54 & \multirow[t]{2}{*}{0.002} \\
\hline$<3.3$ & 74 & 0.84 & \\
\hline \multicolumn{4}{|l|}{ PNI } \\
\hline$\geq 44.8$ & 67 & 0.85 & \multirow[t]{2}{*}{0.021} \\
\hline$<44.8$ & 20 & 0.60 & \\
\hline \multicolumn{4}{|l|}{ Tumor location } \\
\hline Upper & 22 & 0.86 & \multirow[t]{3}{*}{0.047} \\
\hline Middle & 42 & 0.83 & \\
\hline Lower & 23 & 0.65 & \\
\hline \multicolumn{4}{|l|}{ Tumor size } \\
\hline$\geq 20 \mathrm{~mm}$ & 54 & 0.74 & \multirow[t]{2}{*}{0.23} \\
\hline$<20 \mathrm{~mm}$ & 33 & 0.88 & \\
\hline \multicolumn{4}{|l|}{ Macroscopic appearance } \\
\hline Elevated & 46 & 0.89 & \multirow[t]{2}{*}{0.019} \\
\hline Depressed/flat & 41 & 0.68 & \\
\hline \multicolumn{4}{|l|}{ Histology } \\
\hline Differentiated type & 80 & 0.80 & \multirow[t]{2}{*}{0.90} \\
\hline Undifferentiated type & 7 & 0.71 & \\
\hline \multicolumn{4}{|l|}{ Depth of invasion } \\
\hline M/SM1 & 63 & 0.76 & \multirow[t]{2}{*}{0.41} \\
\hline SM2 & 24 & 0.88 & \\
\hline Lymphovascular invasio & & & \\
\hline Present & 26 & 0.77 & 0.85 \\
\hline Absent & 61 & 0.80 & \\
\hline Ulcerative findings & & & \\
\hline Present & 18 & 0.89 & 0.96 \\
\hline Absent & 69 & 0.77 & \\
\hline Additional gastrectomy & & & \\
\hline Yes & 26 & 0.85 & 0.34 \\
\hline No & 61 & 0.77 & \\
\hline
\end{tabular}

OS overall survival, ECOG PS Eastern Cooperative Oncology Group performance status, GNRI geriatric nutritional risk index, $C C I$ Charlson comorbidity index, NLR neutrophil to lymphocyte ratio, $P N I$ prognostic nutritional index, $M$ mucosa, SMI superficial portion of the submucosa within $500 \mu \mathrm{m}$ from the muscularis mucosae, $S M 2$ deep portion of the submucosa $\geq 500 \mu \mathrm{m}$ from the muscularis mucosae 
Table 4 Multivariate analysis of factors associated with overall survival

\begin{tabular}{llll}
\hline Variable & HR & $95 \%$ CI & $P$ value \\
\hline Age $\geq 79$ years & 1.94 & $0.87-4.35$ & 0.11 \\
ECOG PS $\geq 2$ & 1.95 & $0.82-4.67$ & 0.13 \\
CCI $\geq 3$ & 2.79 & $1.16-6.69$ & 0.021 \\
NLR $\geq 3.3$ & 1.86 & $0.73-4.74$ & 0.19 \\
PNI $\leq 44.8$ & 1.50 & $0.60-3.77$ & 0.39 \\
\hline
\end{tabular}

$H R$ hazard ratio, $C I$ confidence interval, ECOG PS Eastern Cooperative Oncology Group performance status, $C C I$ Charlson comorbidity index, $N L R$ neutrophil to lymphocyte ratio, $P N I$ prognostic nutritional index

\section{Discussion}

Several studies have investigated the clinical outcomes of elderly patients with EGC [8-14]. However, longterm outcomes and prognostic factors in elderly patients with EGC were evaluated in only a few studies [10, 12, 14]. To our knowledge, this is the first study to report the long-term outcomes and prognostic factors of elderly patients with EGC after non-curative ESD. In this study, we included various nutritional factors, such as the GNRI, CCI, NLR, and PNI, as possible prognostic factors. As a result, multivariate analysis revealed a $\mathrm{CCI} \geq 3$ to be an independent prognostic factor for elderly patients with EGC after non-curative ESD.

The CCI is a scoring system for classifying comorbidities that might affect the risk of mortality [23]. It is a simple, readily available method for estimating the risk of death from comorbid diseases. There have been several reports about the usefulness of the CCI as a prognostic factor in nasopharyngeal and gastrointestinal cancers [28-30]. While some have reported the value of the CCI for predicting postoperative complications in gastric cancer [31, 32], only two previous study have evaluated the relationship between the CCI and prognosis in elderly patients with EGC treated with ESD [14, 33].

In this study, only one patient died of gastric cancer among 87 elderly patients with EGC after non-curative ESD during long-term follow-up. Another 26 patients died of causes other than gastric cancer, including cancer of other organs (8 patients), pneumonia (7 patients), cardiovascular disease (6 patients), and others (5 patients). Since most of our patients died of comorbidities other than gastric cancer, the close association between CCI and OS in our patients seems to be reasonable.

The CCI is calculated as the sum of scores defined for several comorbidities [23]. Our present data showed that a CCI $\geq 3$ was an independent prognostic factor in elderly patients aged $\geq 75$ years with EGC after non-curative ESD. Because scoring of the CCI is easily completed with a simple medical interview, this may be an advantage, especially in elderly patients treated with ESD. In contrast, Sekiguchi et al. [14] reported that the PNI was the most significant prognostic factor after ESD in elderly patients (aged $\geq 85$ ) who underwent gastric ESD. More recently, Iwai et al. [33] reported that both CCI and PNI were associated with the survival rate in patients with EGC treated by ESD, regardless of age. In the present study, the PNI was found to be a prognostic factor on univariate analysis, but it was not a statistically significant prognostic factor on multivariate analysis.

To date, several studies have reported clinical outcomes of patients with EGC after non-curative ESD [15-19]. Our recent study showed that age was an independent prognostic factor associated with OS in patients with non-curative

Fig. 2 Overall survival curves for patients in a low-CCI group and a high-CCI group $(P<0.001)$

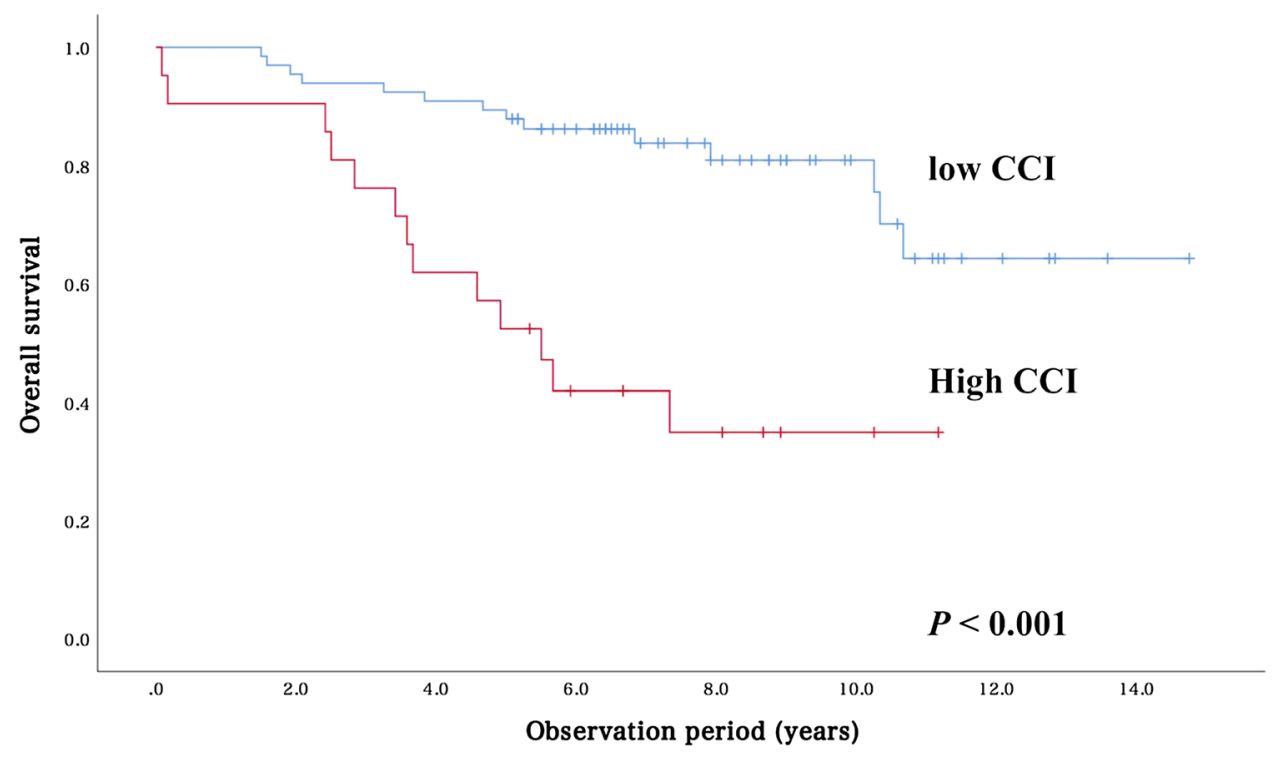


gastric ESD with negative resection margins [18]. Kusano et al. [34] analyzed a much greater number of patients treated by gastric endoscopic resection $>75$ years of age, and found that OS was significantly higher in patients treated by additional surgery than in the other patients. Although the differences were not statistically significant, 5-year OS was higher in our patients with additional gastrectomy than in those without. We thus believe that additional gastrectomy should not be denied in patients with non-curative gastric ESD. However, our results also seem to suggest that careful follow-up without additional gastrectomy may be a choice in elderly patients $\geq 75$ years with high CCI.

Recently, Hatta et al. [35, 36] reported that a risk-scoring system (the eCura system) based on histology of EGC predicted lymph node metastasis (LNM) in patients undergoing radical surgery after non-curative ESD. The system may be useful in decision-making regarding additional treatment after non-curative ESD. However, the value of the eCura system for the prediction of survival in elderly patients with several comorbidities is still unknown. While elderly patients with a low risk of LNM under eCura and a high risk of comorbidities under CCI are candidates for careful follow-up without gastrectomy after non-curative ESD, a stratification system by means of CCI and eCura needs to be elucidated to clarify indications for additional surgery and to predict survival.

The present study has several limitations. First, the retrospective design seems to have introduced selection bias. A prospective study is required to verify the utility of the $\mathrm{CCI}$ as a prognostic factor for elderly patients with EGC after non-curative ESD. Second, the sample size was limited, because this study was conducted at a single center. For example, we were unable to explain why tumor location and macroscopic configuration were found to be significant prognostic factors under univariate analyses. A larger, multicenter cohort study seems to be warranted to confirm our findings.

In conclusion, our study showed that a high CCI was an independent prognostic factor related to poor OS in elderly patients aged $\geq 75$ years with EGC treated with non-curative ESD. On the basis of the results of this study, CCI is suggested to be a marker for decision-making in additional gastrectomy for elderly patients after non-curative ESD.

\section{Compliance with ethical standards}

Conflict of interest The authors declare that they have conflict of interest.

Ethical approval All procedures followed were in accordance with the ethical standards of the responsible committee on human experimentation (institutional and national) and with the Helsinki Declaration of 1964 and later versions. The exemption from informed consent require- ment was permitted by Iwate Medical University Institutional Review Board.

\section{References}

1. Kosaka T, Endo M, Toya Y, et al. Long-term outcomes of endoscopic submucosal dissection for early gastric cancer: a singlecenter retrospective study. Dig Endosc. 2014;26:183-91.

2. Isomoto H, Shikuwa S, Yamaguchi N, et al. Endoscopic submucosal dissection for early gastric cancer: a large-scale feasibility study. Gut. 2009;58:331-6.

3. Toyonaga T, Man-i M, East JE, et al. 1,635 Endoscopic submucosal dissection cases in the esophagus, stomach, and colorectum: complication rates and long-term outcomes. Surg Endosc. 2013;27:1000-8.

4. Oda I, Oyama T, Abe S, et al. Preliminary results of multicenter questionnaire study on long-term outcomes of curative endoscopic submucosal dissection for early gastric cancer. Dig Endosc. 2014;26:214-9.

5. Tanabe S, Ishido K, Matsumoto T, et al. Long-term outcomes of endoscopic submucosal dissection for early gastric cancer: a multicenter collaborative study. Gastric Cancer. 2017;20:45-52.

6. Cho JH, Cha SW, Kim HG, et al. Long-term outcomes of endoscopic submucosal dissection for early gastric cancer: a comparison study to surgery using propensity score-matched analysis. Surg Endosc. 2016;30:3762-73.

7. Park CH, Lee H, Kim D, et al. Clinical safety of endoscopic submucosal dissection compared with surgery in elderly patients with early gastric cancer: a propensity-matched analysis. Gastrointest Endosc. 2014;80:599-609.

8. Kakusima N, Fujishiro M, Kodashima S, et al. Technical feasibility of endoscopic submucosal dissection for gastric neoplasms in the elderly Japanese population. J Gastroenterol Hepatol. 2007;22:311-4.

9. Abe N, Gotoda T, Hirasawa T, et al. Multicenter study of the longterm out comes of endoscopic submucosal dissection for early gastric cancer in patients 80 years of age or older. Gastric Cancer. 2011;15:70-5.

10. Yoshifuku Y, Oka S, Tanaka S, et al. Long-term prognosis after endoscopic submucosal dissection for early gastric cancer in super-elderly patients. Surg Endosc. 2016;30:4321-9.

11. Kato M, Michida T, Kusakabe A, et al. Safety and short-term outcomes of endoscopic submucosal dissection for early gastric cancer in elderly patients. Endosc Int Open. 2016;4:E521-6.

12. Watanabe K, Hikichi T, Nakamura J, et al. Endoscopic submucosal dissection for early gastric cancer in very elderly patients age 85 or older. Endosc Int Open. 2017;5:E17-24.

13. Sumiyoshi T, Kondo H, Fujii R, et al. Short- and long-term outcomes of endoscopic submucosal dissection for early gastric cancer in elderly patients aged 75 years and older. Gastric Cancer. 2017;20:489-95.

14. Sekiguchi M, Oda I, Suzuki H, et al. Clinical outcomes and prognostic factors in gastric cancer patients aged $\geq 85$ years undergoing endoscopic submucosal dissection. Gastrointest Endosc. 2017;85:963-72.

15. Oda I, Gotoda T, Sasako M, et al. Treatment strategy after noncurative endoscopic resection of early gastric cancer. Br J Surg. 2008;95:1495-500.

16. Kang MS, Hong SJ, Kim DY, et al. Long-term outcome after endoscopic submucosal dissection for early gastric cancer: focusing on a group beyond the expanded indication. J Dig Dis. 2015;16:7-13. 
17. Hoteya S, Iizuka T, Kikuchi D, et al. Clinicopathological outcomes of patients with early gastric cancer after non-curative endoscopic submucosal dissection. Digestion. 2016;93:53-8.

18. Toya Y, Endo M, Nakamura S, et al. Clinical outcomes of noncurative endoscopic submucosal dissection with negative resected margins for gastric cancer. Gastrointest Endosc. 2017;85:1218-24.

19. Hatta $\mathrm{W}$, Gotoda $\mathrm{T}$, Oyama $\mathrm{T}$, et al. Is radical surgery necessary in all patients who do not meet the curative criteria for endoscopic submucosal dissection in early gastric cancer? A multi-center retrospective study in Japan. J Gastroenterol. 2017;52:175-84.

20. Oken MM, Creech RH, Tormey DC, et al. Toxicity and response criteria of the Eastern Cooperative Oncology Group. Am J Clin Oncol. 1982;5:649-55.

21. Bouillanne O, Morineau G, Dupont C, et al. Geriatric Nutritional Risk Index: a new index for evaluating at-risk elderly medical patients. Am L Clin Nutr. 2005;82:777-83.

22. Yamada K, Furuya R, Takita T, et al. Simplified nutritional screening tools for patients on maintenance hemodialysis. Am J Clin Nutr. 2008;87:106-13.

23. Charlson ME, Pompei P, Ales KL, et al. A new method of classifying prognostic comorbidity in longitudinal studies: development and validation. J Chronic Dis. 1987;40:373-83.

24. Hirashima M, Higuchi S, Sakamoto K, et al. The ratio of neutrophils to lymphocytes and the phenotypes of neutrophils in patients with early gastric cancer. J Cancer Res Clin Oncol. 1998;124:329-4.

25. Onodera T, Goseki N, Kosaki G. Prognostic nutritional index in gastrointestinal surgery of malnourished cancer patients [in Japanese with an English abstract]. Nihon Geka Gakkai Zasshi. 1984;85:1001-5.

26. Japanese Gastric Cancer Association. Japanese gastric cancer treatment guidelines 2014 (ver.4). Gastric Cancer. 2017;1:1-19.

27. Ono H, Yao K, Fujishiro M, et al. Guidelines for endoscopic submucosal dissection and endoscopic mucosal resection for early gastric cancer. Dig Endosc. 2016;28:3-15.

28. Huang Y, Chen W, Haque W, et al. The impact of comorbidity on overall survival in elderly nasopharyngeal carcinoma patients: a
National Cancer Data Base analysis. Cancer Med. 2018;7:1093101 (Epub ahead of print).

29. Yamashita K, Watanabe M, Mine S, et al. The impact of the Charlson comorbidity index on the prognosis of esophageal cancer patients who underwent esophagectomy with curative intent. Surg Today. 2018;6:632-39 (Epub ahead of print).

30. Tominaga T, Nonaka T, Takeshita H, et al. The Charlson comorbidity index as an independent prognostic factor in older colorectal cancer patients. Indian J Surg. 2018;80:54-60.

31. Huang CM, Tu RH, Lin JX, et al. A scoring system to predict the risk of postoperative complications after laparoscopic gastrectomy for gastric cancer based on a large-scale retrospective study. Medicine. 2015;94:e812.

32. Fujisaki M, Shinohara T, Hanyu N, et al. Laparoscopic gastrectomy for gastric cancer in the elderly patients. Surg Endosc. 2016;30:1380-7.

33. Iwai N, Dohi O, Naito Y, et al. Impact of the Charlson comorbidity index and prognostic nutritional index on prognosis in patients with early gastric cancer after endoscopic submucosal dissection. Dig Endosc. 2018;30:616-23.

34. Kusano C, Iwasaki M, Kaltenbach T, et al. Should elderly patients undergo additional surgery after non-curative endoscopic resection for early gastric cancer? Long-term comparative outcomes. Am J Gastroenterol. 2011;106:1064-9.

35. Hatta W, Gotoda T, Oyama T, et al. A scoring system of stratify curability after endoscopic submucosal dissection for early gastric cancer; "eCura system". Am J Gastroenterol. 2017;112:874-81.

36. Hatta W, Gotoda T, Oyama T, et al. Is the e Cura system useful for selecting patients who require radical surgery after noncurative endoscopic submucosal dissection for early gastric cancer? A comparative study. Gastric Cancer. 2018;21:481-9.

Publisher's Note Springer Nature remains neutral with regard to jurisdictional claims in published maps and institutional affiliations. 